\title{
Benzidamina nelle vulvovaginiti: lo stato dell'arte
}

\section{Secondo Guaschino}

Professore ordinario di Ostetricia e Ginecologia

\section{FISIOLOGIA VAGINALE E IMPORTANZA DEL MICROBIOTA}

Il microhabitat vaginale costituisce un ecosistema complesso governato dall'equilibrio dinamico che caratterizza l'ectosimbiosi mutualistica tra l'ospite e la popolazione microbica autoctona [1].

La microflora vaginale tipica di una donna asintomatica sana comprende un'ampia varietà di generi e specie batteriche anaerobie e aerobie (in un rapporto compreso tra 2:1 e 5:1 [2]) dominate dal genere facoltativo, microaerofilo e anaerobio Lactobacillus $\left(10^{6}-10^{8} \mathrm{CFU} / \mathrm{g}\right)$. Gli altri batteri residenti, tra cui specie streptococciche, batteri gram-negativi, Gardnerella vaginalis, anaerobi, nonché Candida albicans come agente commensale nel 10-25\% delle donne asintomatiche, sono riscontrabili in concentrazioni molto inferiori (Tabella 1) [3-4].

Le complesse interazioni tra l'ospite e la flora lattobacillare contribuiscono alla fisiologia vaginale, all'o-

\section{Indirizzo per la corrispondenza:}

Secondo Guaschino

e-mail: s.guaschino@gmail.com

Accettato: 11 aprile 2017 - Pubblicato online: 28 aprile 2017

(C) 2017 The Authors. This article is published by HPS Srl and licensed under Creative Commons Attribution-NC-ND 4.0 International (CC BY-NC-ND 4.0). Any commercial use is not permitted and is subject to Publisher's permissions. Full information is available at www.aboutpharma.com/publishing/riviste/aboutopen/
Tabella 1. Flora vaginale tipica di una donna sana in età fertile, suddivisa per generi (modificata da [4]).

\begin{tabular}{|c|c|}
\hline $\begin{array}{l}\text { Cocco e bacilli gram (+) } \\
\text { anaerobi aerotolleranti }\end{array}$ & $\begin{array}{l}\text { Lactobacillus } \\
\text { Streptococcus }\end{array}$ \\
\hline $\begin{array}{l}\text { Cocco e bacilli gram }(+) \\
\text { anaerobi facoltativi }\end{array}$ & \multirow{2}{*}{$\begin{array}{l}\text { Corynebacterium } \\
\text { Gardnerella } \\
\text { Staphylococcus } \\
\text { (principalmente S. } \\
\text { epidermidis) } \\
\text { Escherichia } \\
\text { Klebsiella } \\
\text { Proteus }\end{array}$} \\
\hline $\begin{array}{l}\text { Bacilli gram }(-) \\
\text { anaerobi facoltativi }\end{array}$ & \\
\hline Micoplasmi & $\begin{array}{l}\text { Mycoplasma } \\
\text { (soprattutto M. bominis) }\end{array}$ \\
\hline $\begin{array}{l}\text { Bacilli e cocco gram (+) } \\
\text { anaerobi stretti }\end{array}$ & $\begin{array}{l}\text { Atopobium } \\
\text { Peptococcus } \\
\text { Peptostreptococcus } \\
\text { Clostridium } \\
\text { Bifidobacterium } \\
\text { Propionibacterium } \\
\text { Eubacterium }\end{array}$ \\
\hline $\begin{array}{l}\text { Bacilli e cocco gram (-) } \\
\text { anaerobi stretti }\end{array}$ & $\begin{array}{l}\text { Bacteroides } \\
\text { Prevotella }\end{array}$ \\
\hline
\end{tabular}

meostasi e al mantenimento del microbiota vaginale normale, prevenendo la proliferazione di organismi patogeni e opportunisti responsabili di infezioni delle vie genito-urinarie inferiori della donna [5-7].

I meccanismi adottati dai lattobacilli a protezione e regolazione del milieu vaginale sono basati sulle 
proprietà antagoniste da essi esercitate e sono reciprocamente complementari.

In particolare, prevedono:

- l'adesione specifica all'epitelio vaginale, con conseguente formazione di una biopellicola che impedisce la colonizzazione dei patogeni;

- la produzione di composti antimicrobici, che sopprimono la crescita dei patogeni;

- la co-aggregazione con i patogeni, che ne inibisce la moltiplicazione $[4,8]$.

La colonizzazione dei patogeni è impedita dai legami specifici recettoriali e da legami aspecifici (sostenuti da forze elettrostatiche) che i lattobacilli instaurano con le cellule della mucosa vaginale, sottraendo, di conseguenza, siti di legame liberi per l'adesione di altri microrganismi. I lattobacilli, inoltre, possono creare legami specifici con la fibronectina che compone il fluido vaginale e, secernendo biosurfattanti, impedire ai patogeni di aderire alla mucosa [8].

L'inibizione della crescita dei patogeni avviene:

- attraverso la sintesi e la secrezione di sostanze antimicrobiche, tra cui il perossido di idrogeno, che esercita un effetto battericida su alcuni ceppi batterici, le batteriocine, dotate di proprietà antibiotiche e i biosurfattanti;

- sottraendo nutrienti essenziali agli organismi patogeni, come l'arginina deaminasi, e generando, quindi, una competizione per le fonti nutrizionali;

- producendo acido lattico a partire dai carboidrati, così da conferire alla vagina un $\mathrm{pH}$ acido. Sotto l'influenza degli estrogeni, infatti, l'epitelio vaginale produce glicogeno, che Lactobacillus spp è in grado di degradare a glucosio e successivamente ad acido lattico, contribuendo a mantenere il $\mathrm{pH}$ vaginale tipicamente tra 3,8 e 4,5: il basso $\mathrm{pH}$ vaginale è considerato uno dei meccanismi di protezione più efficaci attuato dai lattobacilli nei confronti delle infezioni vaginali $[3,8,9]$.

La co-aggregazione dei lattobacilli con alcuni ceppi batterici patogeni viene favorita dal rilascio di aggregation promoting factor (APF) ed è tale da contrastare la proliferazione dei microrganismi nocivi [8].

La composizione del microbiota vaginale e i rapporti quali-quantitativi dei microrganismi residenti variano anche in funzione dell'età e dell'attività ormonale della donna, con la gravidanza e la fase del ciclo mestruale (Tabella 2) [9-10].

La vagina, infine, rappresenta la via di accesso per diversi agenti infettivi che colonizzano la mucosa vaginale in condizioni locali favorevoli, causando processi infiammatori cronici e disabilitanti [11] Nonostante gli efficienti meccanismi di protezione dell'ecosistema vaginale, la flora lattobacillare può essere sostituita da microrganismi patogeni, condizione associata allo sviluppo di vaginosi batterica, tricomoniasi e altre infezioni del tratto genito-urinario inferiore [4]. Ulteriori fattori esogeni implicati nelle alterazioni dell'equilibro microbiologico vaginale e favorenti la penetrazione e la colonizzazione di agenti patogeni sono rappresentati da tutti gli stati cronicamente debilitanti, dall'immunodepressione e da sindromi dismetaboliche carenziali [12]. Il viraggio in senso patologico dell'ecosistema vaginale può essere inoltre determinato dall'assunzione di farmaci

Tabella 2. Fluttuazioni dinamiche dell'ecosistema vaginale correlate all'età e all'attività ormonale associata (modificata da [10]).

\begin{tabular}{|l|c|c|c|c|c|}
\hline Età & Estrogeni & Epitelio & Glicogeno & pH & Microbiota \\
\hline Neonatale & +++ & & + & 4,5 & Lattobacilli \\
\hline Prepubere & + & & - & 7 & Coliformi \\
\hline Fertile & ++++ & - & ++ & $3,5-4$ & Lattobacilli \\
\hline Menopausale & + & & - & 7 & Coliformi \\
\hline
\end{tabular}


antibiotici, dai trattamenti ormonali, dalle abitudini sessuali e da scarsa o eccessiva igiene intima $[8,13]$. È ormai ampiamente dimostrato che una profonda alterazione dell'ecosistema vaginale costituisce un importante fattore di rischio per l'acquisizione di agenti patogeni virali e batterici a trasmissione sessuale, che condizionano pesantemente lo stato di salute. Tale alterazione, infatti, comunque si sia determinata, favorisce le infezioni da HIV, HPV e Chlamydia trachomatis, agenti responsabili rispettivamente di AIDS, cervicocarcinoma e sterilità.

\section{LE VULVOVAGINITI}

Tra le patologie dell'apparato riproduttore femminile, le vulvovaginiti si distinguono per l'elevata diffusione e frequenza. Negli Stati Uniti si è calcolato che siano più di 6 milioni all'anno le consultazioni mediche, con una spesa annuale complessiva superiore a un miliardo di dollari, per le visite ginecologiche e farmaci da automedicazione.
Le vulvovaginiti possono essere considerate una vera e propria patologia sociale in quanto coinvolgono la sfera privata della paziente, compromettendo la gestione della propria sessualità e creando uno stato di disagio nelle sue relazioni, sia con la cerchia sociale sia con il medico $[3,14]$.

Il $90 \%$ dei casi di vaginite è riconducibile a un'infezione microbica, di cui il 60\% di origine batterica, il $20 \%$ di natura micotica e il $10 \%$ da Trichomonas vaginalis. Il rimanente $10 \%$ è determinato da fenomeni a eziologia non infettiva, quali reazioni irritative, variazioni ormonali e altre condizioni che contribuiscono ad alterare l'ecosistema vaginale (Tabella 3) [1,15]. La vulvovaginite è favorita da modifiche indotte del $\mathrm{pH}$ locale, da condizioni patologiche concomitanti sia locali sia sistemiche e da terapie che modificano la composizione del microbiota. Tutti questi fattori contribuiscono a rendere l'ambiente vaginale più vulnerabile alla proliferazione dei microrganismi patogeni e allo svilupparsi di episodi infettivi [14].

Tabella 3. Eziologia delle vulvovaginiti (modificata da [15]).

\begin{tabular}{|c|c|}
\hline Tipo di vulvovaginite & Cause \\
\hline Vaginosi batterica & $\begin{array}{l}\text { Gardnerella vaginalis, Mycoplasma hominis, specie } \\
\text { di Mobiluncus, specie di Bacteroides (a esclusione di } \\
\text { Bacteroides fragilis) }\end{array}$ \\
\hline Vaginite aerobia & Streptococco beta-emolitico, Escherichia coli \\
\hline Candidosi vulvovaginale & $\begin{array}{l}\text { Candida albicans, Candida glabrata, Candida } \\
\text { tropicalis, Candida krusei }\end{array}$ \\
\hline Tricomoniasi & Trichomonas vaginalis \\
\hline Vaginite atrofica & Carenza estrogenica \\
\hline Irritazione da agenti chimici & $\begin{array}{l}\text { Saponi, prodotti per l'igiene intima (tamponi, assorbenti } \\
\text { intimi, profilattici in lattice) }\end{array}$ \\
\hline Lichen planus (di tipo desquamativo) & $\begin{array}{l}\text { Lesioni ipercheratinizzate piatte pruriginose o dolenti; } \\
\text { associato a lesioni vulvari e orali }\end{array}$ \\
\hline Vaginite allergica & $\begin{array}{l}\text { Sperma, irrigazioni vaginali, prodotti per l'igiene intima, } \\
\text { tamponi, assorbenti intimi, profilattici in lattice o } \\
\text { diaframmi, coloranti, inalazione di allergeni, esposizioni } \\
\text { occupazionali }\end{array}$ \\
\hline Corpo estraneo con o senza infezione o trauma & $\begin{array}{l}\text { Tamponi, dispositivi per la contraccezione, ovuli } \\
\text { vaginali, altro }\end{array}$ \\
\hline
\end{tabular}




\section{VULVOVAGINITI A EZIOLOGIA INFEITIVA}

\section{Vaginosi batterica}

La vaginosi batterica non è causata da patogeni specifici, ma è caratterizzata dalla crescita prevalente di microrganismi anaerobi (G. vaginalis, Prevotella spp, M. hominis, Mobiluncus spp) e dalla concomitante riduzione della componente lattobacillare con conseguente aumento del $\mathrm{pH}$ da $<4,5$ fino a 7 . La vaginosi batterica si configura quindi come un'alterazione dell'equilibrio dell'ecosistema vaginale; il quadro clinico è molto variabile, potendo essere anche asintomatica. È caratterizzata dall'assenza di segni di infiammazione e dalla presenza di secrezioni vaginali grigio-biancastre, di odore amminico. Non può essere considerata una malattia sessualmente trasmissibile, anche se sono descritti casi di trasmissione orogenitale in donne omosessuali. Il trattamento è eziologico, con un tasso di recidive pari al 30\% entro 1 anno [3,14,15].

\section{Vaginite aerobia}

La vaginite aerobia è caratterizzata, come la vaginosi batterica, da un'alterazione dell'ecosistema vaginale con netta riduzione della popolazione lattobacilla- re. Si differenzia per la presenza di un importante stato infiammatorio delle mucose che condiziona la sintomatologia sia soggettiva, sia oggettiva. Il bruciore intenso con leucorrea caratterizzata da odore acre e pungente, la dispareunia e l'iperemia delle mucose sono i sintomi più frequenti. La diagnosi è possibile solamente con la batterioscopia a fresco, che consente di evidenziare un tappeto di leucociti citotossici associati a cellule vaginali dello strato basale. I lattobacilli sono per lo più assenti. Ad oggi non esiste una terapia standard codificata, anche se clindamicina, cloramfenicolo e kanamicina sembrano essere gli antibiotici più efficaci. In tali casi l'associazione con un antinfiammatorio locale, quale benzidamina, è di sicura utilità nel ridurre rapidamente la sintomatologia, dati i suoi effetti antinfiammatorio e analgesico.

\section{Candidosi vulvovaginale - Tricomoniasi}

I patogeni che causano questo tipo di vaginite sono rispettivamente Candida, più frequentemente albicans, e Tricomonas vaginalis, protozoo trasmesso per via sessuale. In entrambi i casi la componente infiammatoria è prevalente, con sintomi evidenti quali prurito,

Tabella 4. Caratteristiche tipiche delle vulvovaginiti causate da microrganismi infettivi (modificata da $[3,14]$ ).

\begin{tabular}{|c|c|c|c|c|c|c|}
\hline Infezione & Sintomi e segni & $\begin{array}{l}\text { Esame } \\
\text { obiettivo }\end{array}$ & Secrezioni & Odore & pH & Striscio vaginale \\
\hline $\begin{array}{l}\text { Vaginosi } \\
\text { batterica }\end{array}$ & $\begin{array}{l}\text { Variabili, nel 50\% } \\
\text { asintomatica }\end{array}$ & & $\begin{array}{l}\text { Omogenee di } \\
\text { colore dal bianco } \\
\text { al grigio, talvolta } \\
\text { schiumose }\end{array}$ & $\begin{array}{l}\text { Presente } \\
\text { (amminico) }\end{array}$ & $>4,5$ & $\begin{array}{l}\text { >20\% di clue cell } \\
\text { Odore amminico } \\
\text { con aggiunta } \\
\text { di idrossido di } \\
\text { potassio }\end{array}$ \\
\hline $\begin{array}{l}\text { Vaginite } \\
\text { aerobia }\end{array}$ & $\begin{array}{l}\text { Bruciore, } \\
\text { dispareunia }\end{array}$ & $\begin{array}{l}\text { Edema, } \\
\text { iperemia }\end{array}$ & $\begin{array}{l}\text { Omogenee } \\
\text { bianco-grigiastre }\end{array}$ & $\begin{array}{l}\text { Presente } \\
\text { (acre) }\end{array}$ & $>4,5$ & $\begin{array}{l}\text { Batteri, } \\
\text { polimorfonucleati, } \\
\text { cellule basali }\end{array}$ \\
\hline $\begin{array}{l}\text { Candidosi } \\
\text { vulvovaginale }\end{array}$ & $\begin{array}{l}\text { Prurito, disuria, } \\
\text { bruciore }\end{array}$ & $\begin{array}{l}\text { Fissurazioni, } \\
\text { eritema } \\
\text { vaginale }\end{array}$ & $\begin{array}{l}\text { Consistenti, di } \\
\text { colore dal bianco } \\
\text { al giallo, di } \\
\text { aspetto caseoso }\end{array}$ & Assente & $<4,5$ & Ife o spore \\
\hline Tricomoniasi & $\begin{array}{l}\text { Prurito, } \\
\text { disuria, spesso } \\
\text { asintomatica }\end{array}$ & $\begin{array}{l}\text { "Cervice a } \\
\text { fragola" }\end{array}$ & $\begin{array}{l}\text { Profuse, verdi, } \\
\text { schiumose }\end{array}$ & $\begin{array}{l}\text { Presente o } \\
\text { assente }\end{array}$ & $>4,5$ & $\begin{array}{l}\text { Tricomonadi } \\
\text { mobili e maggior } \\
\text { numero di leucocit }\end{array}$ \\
\hline
\end{tabular}


bruciore e disuria (Tabella 4) [3,14,15]. Il trattamento è eziologico e risolutivo nella quasi totalità dei casi. Tuttavia, data la forte componente infiammatoria e la sintomatologia invalidante, è consigliabile associare un trattamento antinfiammatorio [16]. Benzidamina in associazione a trattamento eziologico nelle infezioni da Candida e da T. vaginalis può considerarsi un efficace approccio terapeutico nel trattamento della sintomatologia infiammatoria, senza interferenza negativa sull'ecosistema vaginale. Inoltre, il sollievo sintomatologico precoce che ne deriva può migliorare la compliance delle pazienti al trattamento eziologico.

\section{VULVOVAGINITI AD EZIOLOGIA NON INFEITIVA}

\section{Vaginite atrofica}

L'atrofia vulvovaginale è caratteristica del periodo post-menopausale, in cui la riduzione dei livelli di estrogeni influenza la degenerazione dei tessuti, con conseguenti modifiche biochimiche e morfologiche dell'ambiente vaginale; essa costituisce parte integrante della sindrome genito-urinaria menopausale. La riduzione dei livelli estrogenici provoca assottigliamento graduale dell'epitelio vaginale, diminuzione della produzione di glicogeno e riduzione e modifica della componente lattobacillare, con conseguente alcalinizzazione dell'ambiente. La composizione del microbiota si modifica con prevalenza di microrganismi di origine enterica, che possono favorire l'insorgenza di processi infiammatori e infettivi a livello vulvovaginale e urinario [16,17].

\section{Radiomucosite vaginale}

L'irradiazione della regione pelvica è comunemente praticata per il trattamento dei tumori della cervice e del colon-retto con effetti curativi elevati, ma anche con complicanze indesiderate, immediate e tardive, a carico della mucosa vaginale. Il rivestimento epiteliale viene lesionato e compare un'infiammazione estesa, che può favorire l'insorgenza di infezioni ed emorragie [18,19].

\section{PROPRIETÀ FISICO-CHIMICHE E ATTIVITÀ FARMACOLOGICHE DI BENZIDAMINA}

Benzidamina, o N,N-dimetil-3-[[1-(fenilmetil)-1H-indazolo-3-yl]ossi]-1-propanamina (Figura 1) [20], è stata sintetizzata negli anni Sessanta nei laboratori di ricerca Angelini.

Benzidamina è un farmaco somministrato per via topica, dotato di attività anestetica, analgesica, antinfiammatoria e antimicrobica. L'insieme di tali proprietà la rende uno strumento terapeutico importante nel trattamento delle vulvovaginiti caratterizzate da piccole perdite vaginali, prurito, bruciore, infiammazione e dolore vulvare. Benzidamina viene utilizzata anche per l'igiene intima durante il puerperio [21].

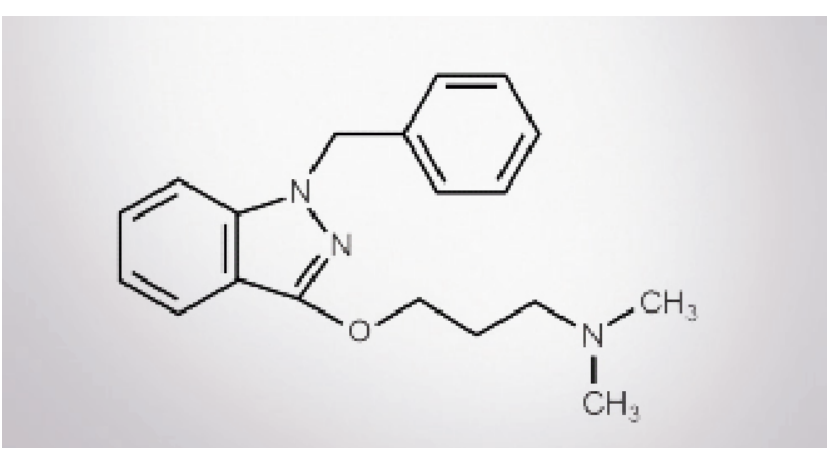

Figura 1. Struttura chimica di benzidamina (modificata da [20]).

\section{MECCANISMO D'AZIONE}

Benzidamina associa all'attività antinfiammatoria un effetto anestetico di superficie e un'azione antisettica.

\section{Attività antinfiammatoria}

L'attività antinfiammatoria di benzidamina si esplica a diversi livelli $[21,22]$. Il meccanismo principale si basa su un'inibizione della sintesi delle citochine, che determina una diminuita permeabilità vasale $\mathrm{e}$ un'inibizione della migrazione dei leucociti al sito di infiammazione e della loro degranulazione $[23,24]$. Studi in vitro su monociti circolanti stimolati con lipopolisaccaridi (LPS) o C. albicans, hanno dimostrato che benzidamina agisce come potente soppressore del tumor necrosis factor (TNF)- $\alpha$ e in minor misura di interleuchina (IL)-1 $\beta$ e di monocyte chemotactic 
protein (MCP)-1/CCL2, noti per essere potenti mediatori dell'infiammazione [23]. Infatti, come riportato in letteratura, TNF- $\alpha$ e IL-1 $\beta$ possiedono un ruolo importante nel meccanismo molecolare di infiammazione in pazienti con vulvovaginiti da Candida, mentre MCP-1 è coinvolto nel reclutamento dei leucociti nelle secrezioni vaginali nelle pazienti con vaginosi batterica e vulvovaginiti da Candida [25]. Inoltre, esperimenti in vitro con monociti umani isolati hanno evidenziato che benzidamina inibisce la protein-kinasi attivata dai mitogeni, fattore coinvolto nel processo di sintesi delle citochine proinfiammatorie e nella transduzione del segnale associata alla migrazione cellulare. Questo contribuisce al limitato richiamo di leucociti nel sito di infiammazione e di conseguenza all'attività antinfiammatoria del farmaco [26]. Il trattamento in vivo con benzidamina ( $40 \mathrm{mg} / \mathrm{kg} \mathrm{sc}$ ) ha mostrato di proteggere i topi dalla mortalità indotta dai LPS, con marcata riduzione di TNF- $\alpha$ e IL- $1 \beta$ nel siero, confermando in vivo la capacità antinfiammatoria di benzidamina [23].

\section{Attività analgesica e anestetica}

Nell'utilizzo topico benzidamina ha dimostrato di avere attività anestetica locale, bloccando i canali cationici coinvolti nella trasmissione del dolore. Infatti è noto che in molti anestetici locali l'attività è correlata a un'interazione con i canali cationici. Benzidamina ha mostrato un analogo tipo di attività inibendo il flusso di corrente nei canali del sodio [27]. Utilizzando omogenati di corteccia cerebrale di ratto e batracotossina marcata come radioligante specifico, benzidamina ha mostrato affinità per il canale con un $\mathrm{IC}_{50} \mathrm{di} 7 \mu \mathrm{M}$. Tale interazione è stata confermata a livello funzionale in neuroni deputati alla percezione del dolore (nocicettori). In tale modello si evidenzia che benzidamina è in grado di attenuare l'eccitabilità dei nocicettori attraverso un blocco dei canali del sodio, a dimostrazione dell'attività anestetica locale [27].

\section{Attività antibatterica e antifungina}

L'attività antibatterica e antifungina di benzidamina è stata evidenziata nel passato [28]. In studi più recenti sulle specie di Candida, benzidamina a bassi dosaggi mostra un'attività fungistatica, con valori di concentrazione minima inibente tra 6,25 e $50 \mu \mathrm{g} / \mathrm{mL}$, dovuta probabilmente a un danno metabolico dei miceti; mentre a concentrazioni maggiori $(200 \mu \mathrm{g} / \mathrm{mL})$ manifesta attività antimicotica dovuta alla lesione diretta della membrana plasmatica [29]. È stato evidenziato che valori di $\mathrm{pH}$ prossimi alla neutro-alcalinità, caratteristici di molte infezioni vulvovaginali, favoriscono l'azione di benzidamina, consentendo un'antisepsi mirata della flora patogena nel rispetto di quella lattobacillare (acidofila). Questa particolare caratteristica permette di contrastare la crescita della flora patogena salvando la flora saprofita e ristabilendo l'omeostasi dell'ecosistema vaginale $[20,28,30]$. Il ricorso agli antisettici, soprattutto nelle forme polimicrobiche, è oggi particolarmente consigliato per ridurre all'indispensabile l'uso di antibiotici, contro i quali diverse specie batteriche e fungine hanno sviluppato resistenze difficilmente superabili.

\section{CENNI DI FARMACOCINETICA}

In genere benzidamina non è ben assorbita attraverso la cute e le mucose, producendo livelli plasmatici trascurabili, insufficienti per un effetto farmacologico sistemico. In uno studio clinico farmacocinetico in crossover, dopo somministrazione di una dose singola di benzidamina $0,1 \%$ soluzione per lavanda vaginale $(140 \mathrm{mg} /$ dose), è stato ottenuto un picco di concentrazione plasmatica di $9,7 \pm 8,0 \mathrm{ng} / \mathrm{mL}\left(\mathrm{C}_{\max } ;\right.$ media $\left.\pm \mathrm{DS}\right)$ dopo $7,5 \pm 3,2$ ore dalla somministrazione del farmaco $\left(\mathrm{t}_{\max } ;\right.$ media $\left.\pm \mathrm{DS}\right)$. In seguito, la concentrazione media plasmatica diminuisce fino a $3,2 \mathrm{ng} / \mathrm{mL}$ a 30 ore post-dose, e la disponibilità sistemica media di benzidamina è 1,3\% della soluzione per lavanda utilizzata [31]. È interessante notare che la somministrazione topica di benzidamina $0,1 \%$ produce concentrazioni plasmatiche simili nelle pazienti con infiammazione vaginale e senza. Questo indica che l'assorbimento e l'esposizione sistemica al farmaco non sono influenzati significativamente dall'infiammazione della mucosa vaginale [32]. In seguito a somministrazione vaginale benzidamina si concentra nella mucosa [3133]. Indagini fluorimetriche hanno dimostrato che la concentrazione media del farmaco ritrovata nella 
mucosa vaginale dopo somministrazione di soluzione vaginale $0,1 \%$ due volte al giorno per 2 giorni consecutivi (benzidamina totale $560 \mathrm{mg}$ ), è $9,7 \pm 6,2$ $\mu \mathrm{g} / \mathrm{g}$ di tessuto fresco. Questa quantità è maggiore del range di concentrazione antinfiammatoria minima $(2-8 \mu \mathrm{g} / \mathrm{g})$ stabilita negli studi di farmacologia [34]. Benzidamina è metabolizzata nel fegato, come dimostrato da studi in vitro che hanno utilizzato microsomi epatici ed epatociti umani in colture monostratificate $[35,36]$. Studi in vitro hanno evidenziato che benzidamina è metabolizzata nel suo N-ossido da flavine contenenti mono-ossigenasi3 (FMO3). I livelli sistemici di farmaco sono escreti principalmente nelle urine, la maggior parte in forma di metaboliti inattivi, oppure in forma coniugata [33].

\section{EFFICACIA E SICURE77A CLINICA}

\section{Efficacia clinica}

L'efficacia di preparazioni per uso ginecologico di benzidamina è stata dimostrata per più di 50 anni in numerosi studi di farmacologia e clinici, in donne adulte, anche in gravidanza [37,38].

Tali studi dimostrano l'efficacia nel raggiungere una rapida e significativa regressione della sintomatologia (fastidio, irritazione, prurito, arrossamento, bruciore, dolore) e dei segni locali di infiammazione (perdite vaginali, edema, eritema e congestione) associati alle vulvovaginiti a eziologia infettiva e non infettiva.

\section{Benzidamina nella vaginosi batterica}

Benzidamina è stata utilizzata come soluzione 0,1\% per lavanda vaginale e anche nelle altre forme eventualmente disponibili per utilizzo ginecologico, due volte al giorno per 10 giorni, in pazienti con vaginosi batterica, candidosi vulvovaginale e tricomoniasi in numerosi studi clinici [16,39-43].

In particolare uno studio condotto su 14 pazienti affette da vaginosi batterica trattate con benzidamina $0,1 \%$, due volte al giorno per 10 giorni, ha riportato un progressivo e significativo miglioramento della sintomatologia già dopo 4 giorni di trattamento $(-27 \%)$, proseguito con un dimezzamento dello score medio iniziale a 7 giorni $-55,4 \%$ ) e terminato al giorno 10 con una riduzione media percentuale del $74,3 \%$. Il dato microbiologico registrato nelle pazienti in terapia con benzidamina $0,1 \%$ risulta essere particolarmente interessante. Infatti la costante riduzione di G. vaginalis risulta essere correlata all'aumento delle colonie di Lactobacillus acidophilus del 50\% alla fine del trattamento (Figura 2) [30].

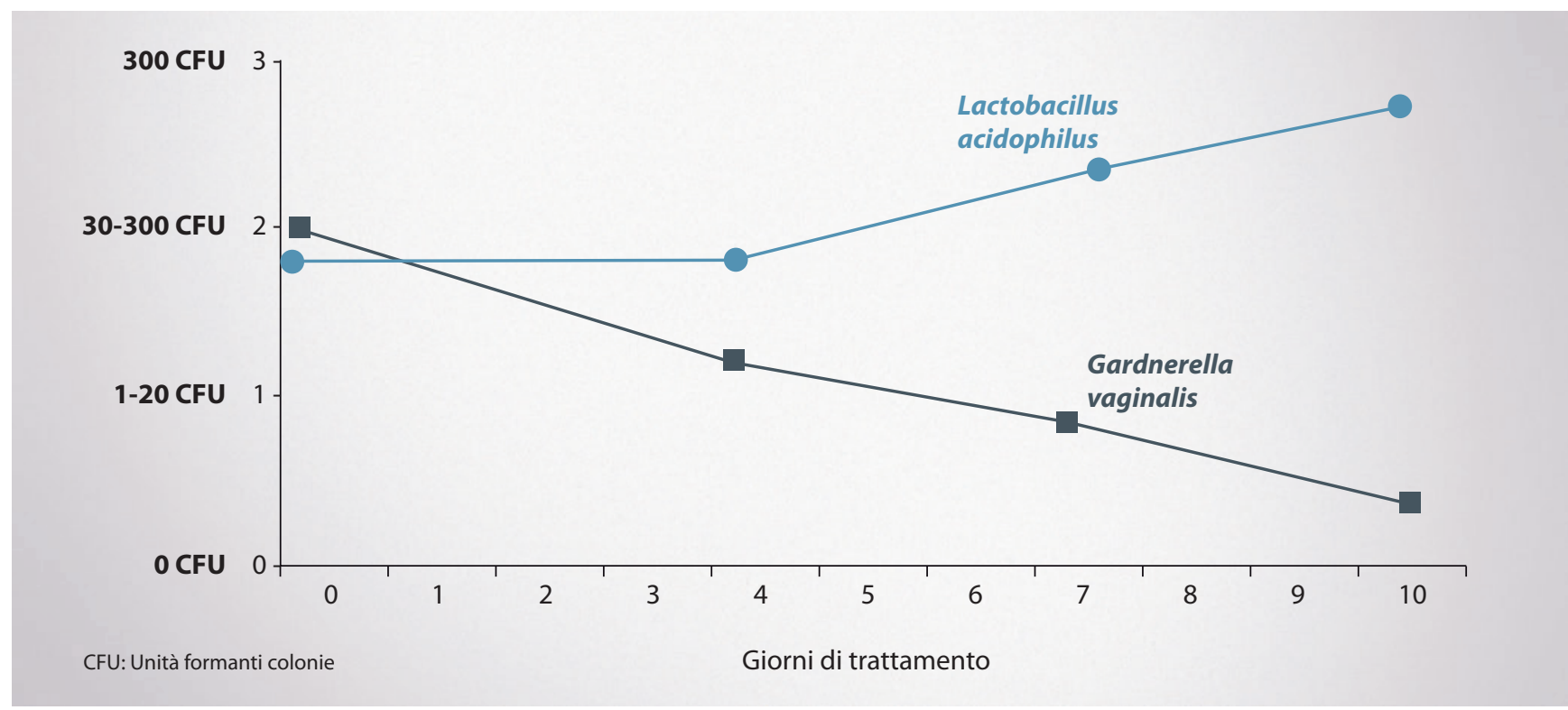

Figura 2. Correlazione tra l'eradicazione di Gardnerella vaginalis e la crescita di Lactobacillus acidophilus in coltura (modificata da [30]). 
In un altro studio clinico di confronto benzidamina lavanda $0,1 \%$ vs placebo, 15 pazienti affette da vaginite ricorrente sono state trattate due volte al giorno per i primi 7 giorni e, per i successivi 3 mesi, con applicazioni bisettimanali.

I risultati evidenziano il buon indice di efficacia terapeutica di benzidamina nel trattamento delle vulvovaginiti aspecifiche recidivanti, con una riduzione del punteggio relativo alla valutazione della sintomatologia e dell'obiettività clinica del 48,7\% dopo i primi 7 giorni e dell' $82,3 \%$ alla fine dei 3 mesi di trattamento con risoluzione del reperto obiettivo pari al $92,5 \%$ al termine del ciclo di terapia; differenze statisticamente significative rispetto al gruppo di controllo. Anche per la riduzione del $\mathrm{pH}$ vaginale si riscontra un significativo miglioramento, con un valore medio finale di $4,7 \pm 0,2$ molto vicino ai valori normali, contro $5,8 \pm 0,2$ con placebo $(\mathrm{p}<0,001)$ [44].

Ulteriori studi hanno confrontato l'efficacia e la tollerabilità di benzidamina $0,1 \%$ con una soluzione a base di iodiopovidone, metronidazolo, acetato di alluminio (soluzione di Burrow) o acido lattico in un ampio numero di pazienti, dimostrando tutti uno spettro d'azione più completo e una più elevata effi- cacia di benzidamina associata a un tasso significativamente minore di effetti collaterali [45-48].

Inoltre nello studio di Boselli e coll. vs iodiopovidone $10 \%$ e vs soluzione di acido lattico, benzidamina è stata l'unico farmaco a determinare un miglioramento statisticamente significativo della sintomatologia soggettiva (Figura 3) e dei segni obiettivi (Figura 4) con, in particolare, una marcata riduzione dei segni infiammatori e del $\mathrm{pH}$ vaginale $(\mathrm{p}<0,001)$ [46].

Il trattamento topico combinato di benzidamina $0,1 \%$ e supposte di metronidazolo in 14 pazienti ha dimostrato un'efficacia sovrapponibile a quella ottenuta con metronizadolo per os, ma senza gli effetti sistemici associati alla terapia orale [48].

\section{Benzidamina nella candidosi vulvovaginale $e$ nella tricomoniasi}

In uno studio randomizzato, in doppio cieco, benzidamina soluzione $0,1 \%$ vs placebo in 75 pazienti affette da candidosi vulvovaginale e tricomoniasi ha determinato una riduzione dei sintomi infiammatori significativamente superiore rispetto a placebo, con una risposta clinica e un effetto analgesico evidente $(\mathrm{p}<0,05)$ sin dal primo giorno di trattamento [49].

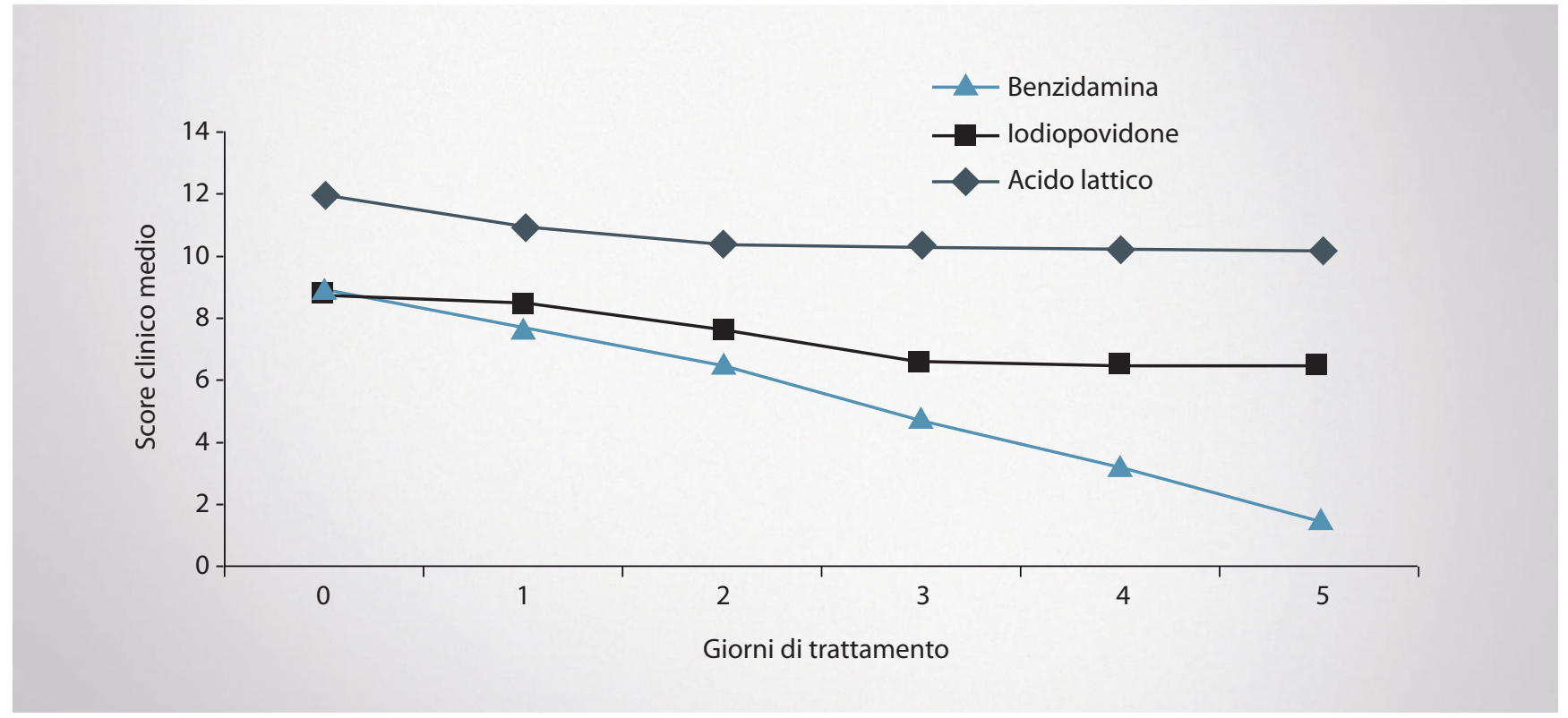

Figura 3. Variazioni nella sintomatologia soggettiva (prurito, tensione, bruciore, dolore, leucorrea) durante il trattamento con benzidamina soluzione $0,1 \%$, iodiopovidone soluzione $10 \%$ e soluzione di acido lattico in pazienti con vaginite aspecifica (modificata da [46]). 
L'efficacia e la rapida risposta contro l'infiammazione vulvovaginale e i sintomi dolorosi sono state dimostrate in ulteriori, numerose indagini cliniche condotte su pazienti affette da candidosi e tricomoniasi: in tutte le sperimentazioni effettuate, già la prima applicazione di benzidamina $0,1 \%$ ha garantito un notevole sollievo dei sintomi e un recupero completo nella maggior parte delle pazienti già dopo 3 applicazioni [16,39,40,42,43].

Ulteriori studi hanno confermato l'elevata efficacia di benzidamina verso placebo [50-52].

\section{Benzidamina nella vaginite atrofica}

In uno studio clinico randomizzato, 64 pazienti affette da vaginite atrofica postmenopausale complicata con sintomi di vaginite sono state trattate con associazione di benzidamina ed estriolo vaginale e 42 pazienti con la sola terapia ormonale, per un numero complessivo di 106 pazienti di età compresa tra 48 e 72 anni. La terapia ormonale integrata con benzidamina è risultata associata a una più precoce risoluzione dei sintomi infiammatori e scomparsa dei batteri dallo striscio citologico $[15,17]$.

\section{Benzidamina nella vulvovaginite da irritanti locali}

La presenza di corpi estranei (tamponi, dispositivi intrauterini, pessari) causa spesso infiammazioni a livello vulvovaginale, con aumentato rischio di infezione.

Uno studio in doppio cieco condotto su 64 donne portatrici di dispositivi intrauterini ha esaminato l'efficacia di benzidamina $0,1 \%$ e dimostrato l'elevato grado di protezione dall'infezione e di prevenzione dell'insorgenza di forme subcliniche di vaginite, con un miglioramento rilevante dei sintomi obiettivi (68\% con benzidamina vs $41 \%$ con placebo) e una conseguente azione preventiva verso patologie pelviche più gravi [53].

Uno studio prospettico su 55 pazienti affette da prolasso uterino sintomatico di vario grado e portatrici di pessario ha valutato l'impiego di benzidamina nel sollievo della sintomatologia e della clinica tipicamente associati alla presenza di corpi estranei intravaginali, evidenziando un miglioramento nel $74 \%$. Benzidamina, somministrata con regolarità, migliorava il fastidio locale, l'edema, l'iperemia, le ulcerazioni della mucosa e le secrezioni vaginali come pure

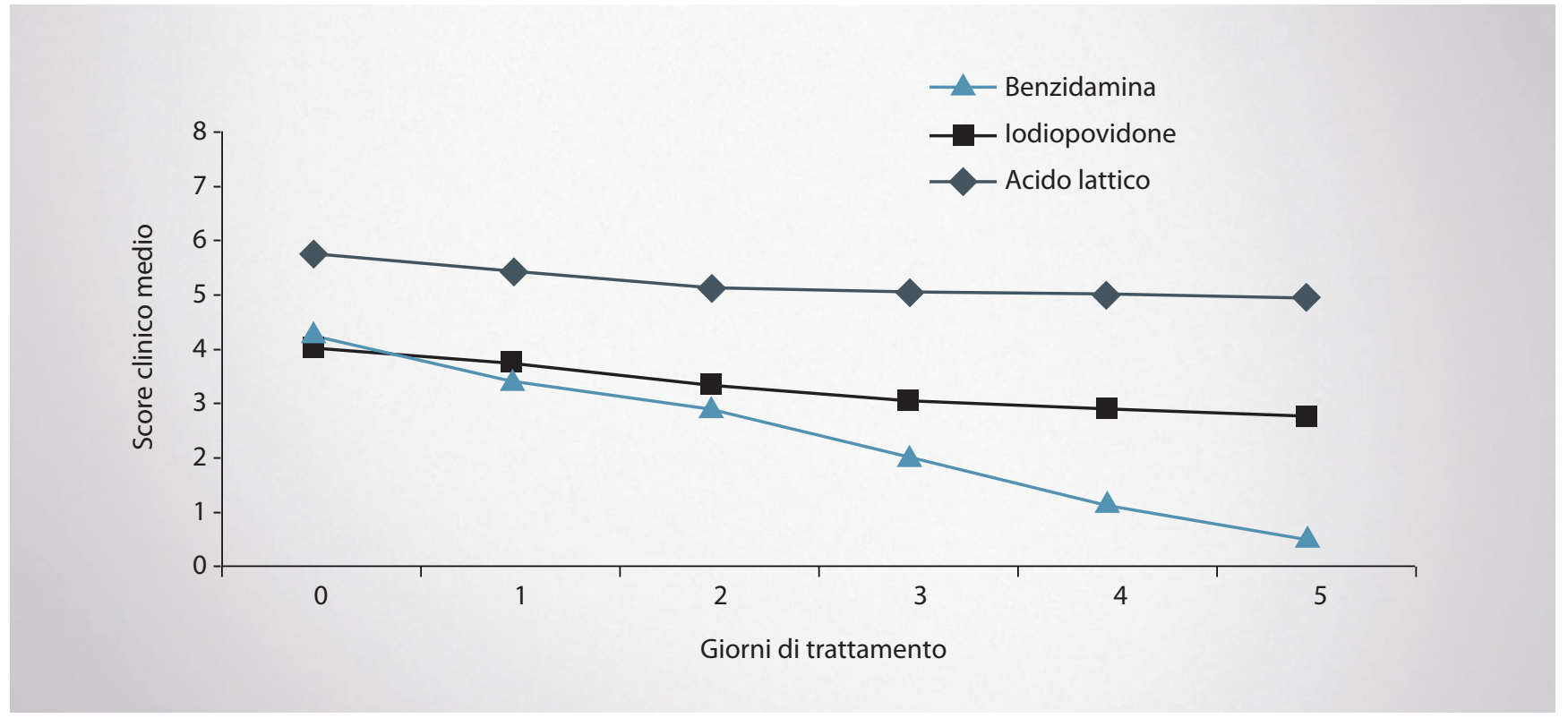

Figura 4. Variazioni nella sintomatologia oggettiva (citologia, colposcopia, esame batterioscopico, pH) durante il trattamento con benzidamina soluzione $0,1 \%$, iodiopovidone soluzione $10 \%$ e soluzione di acido lattico in pazienti con vaginite aspecifica (modificata da [46]). 
i risultati citologici e il grado di soddisfazione delle pazienti esaminate [54].

\section{Benzidamina nella radiomucosite vaginale}

Benzidamina è stata utilizzata in oncologia per il sollievo della sintomatologia post-radioterapia nelle neoplasie dell'area ginecologica.

Per valutare l'attività antinfiammatoria di benzidamina $0,1 \%$, è stato effettuato uno studio clinico randomizzato, in doppio cieco, a gruppi paralleli (benzidamina + conservante, benzidamina e placebo) su 30 pazienti con vaginite secondaria a radioterapia per carcinoma dell'utero. Le pazienti sono state sottoposte a trattamento 12-24 ore dopo la radioterapia due volte al giorno per 15 giorni, dimostrando la superiore efficacia antinfiammatoria di benzidamina già dopo 5 giorni di trattamento [55].

A conferma dell'efficacia in questa categoria di pazienti, un ulteriore studio clinico controllato $v s$ placebo su 32 pazienti ha dimostrato un miglioramento statisticamente significativo nella sintomatologia soggettiva e nell'aspetto della cervice e della vagina alla colposcopia, rispetto a un peggioramento di tutti i parametri clinici riscontrato nel gruppo placebo [18]. In nessuno dei due studi sono stati riscontrati effetti indesiderati, supportando anche sotto questo aspetto il beneficio di benzidamina nel trattamento della radiomucosite vaginale acuta $[19,56]$.

In 15 pazienti sottoposte a chirurgia uterina e ovarica e a irradiazione, benzidamina in soluzione vaginale $0,1 \%$ ha determinato un rapido recupero epiteliale, prevenendo lo sviluppo di lesioni necrotiche serie e garantendo un adeguato trattamento dell'infiammazione delle mucose vaginale e vulvare [57].

\section{Benzidamina in gravidanza}

La somministrazione topica di benzidamina non è controindicata per il trattamento delle vaginiti in gravidanza e durante l'allattamento, in virtù dell'elevata tollerabilità locale e dell'assenza di effetti sistemici. Lo ha dimostrato, tra gli altri, uno studio condotto su 46 donne all' $8^{\mathrm{a}}-12^{\mathrm{a}}$ settimana di gestazione, candidate alla villocentesi e affette da vaginosi batterica, nelle quali benzidamina $0,1 \%$, somministrata una volta al giorno per 10 giorni, ha determinato la regressione della sintomatologia e dell'infezione [38].

\section{Benzidamina nel puerperio}

Benzidamina $0,1 \%$ soluzione vaginale per lavaggio dei genitali esterni è stata utilizzata in uno studio multicentrico condotto su 496 pazienti dopo parto naturale con o senza episiotomia. Le pazienti sono state divise in due gruppi di trattamento: igiene perineale con acqua e sapone + benzidamina $0,1 \%$ lavanda, irrigata nella zona dei genitali esterni due volte al giorno $v s$ igiene perineale con acqua e sapone, per 3-4 giorni. Nel gruppo trattato con benzidamina cloridrato $0,1 \%$ è stata registrata una rapida e definita diminuzione dell'intensità del dolore, della sensazione di bruciore (Figura 5A), del prurito (Figura 5B) e dell'edema rispetto alle pazienti che utilizzavano solamente acqua e sapone per l'igiene intima [58].

\section{Sicurezza di impiego}

Per quanto riguarda il profilo di sicurezza del farmaco, studi clinici e dati di farmacovigilanza post-marketing hanno dimostrato che benzidamina per uso topico vaginale non causa effetti collaterali né eventi avversi clinicamente significativi. L'aggiornamento periodico dei dati di safety [59], che contiene tutti i dati riguardanti benzidamina per utilizzo ginecologico raccolti dal servizio di farmacovigilanza di ACRAF SpA nel periodo 1 novembre 2009-31 ottobre 2012 (1.587.304 pazienti trattate) ha registrato 12 eventi avversi ( 3 seri/inattesi, 8 non seri/inattesi, 1 non seri/ atteso) durante il periodo di tempo descritto. Dieci dei dodici casi erano dovuti all'utilizzo del prodotto tramite un'errata via di sommininistrazione: ingestione orale della polvere per soluzione vaginale. Complessivamente benzidamina per uso vaginale può essere considerata efficace e sicura nel trattamento delle vulvovaginiti di ogni origine e natura caratterizzate da perdite vaginali, irritazione, prurito, bruciore e dolore vulvare.

\section{CONCLUSIONI}

Le vulvovaginiti micotiche, batteriche e protozoarie rappresentano la più frequente causa di ricorso 
alla visita specialistica ginecologica e di utilizzo di farmaci da automedicazione. Una corretta diagnosi elimina i frequenti errori terapeutici e riduce la cronicizzazione dei sintomi. L'utilizzo di farmaci antibiotici e antimicotici non sempre è necessario e utile. Alla luce di quanto riportato in letteratura, l'impiego di antisettici con dimostrate attività antimicrobiche, quali benzidamina, può essere la scelta migliore in molti casi. Tra gli antisettici utilizzabili in campo ginecologico con attività dimostrata, ricordiamo anche dequalinio, clorexidina e iodiopovidone.

La significativa efficacia, l'ottimo profilo di sicurezza e l'alto grado di tollerabilità di benzidamina impiegata nel trattamento delle vulvovaginiti, sono tali da determinare un grado di soddisfazione e un livello di adesione alla terapia estremamente elevati, grazie

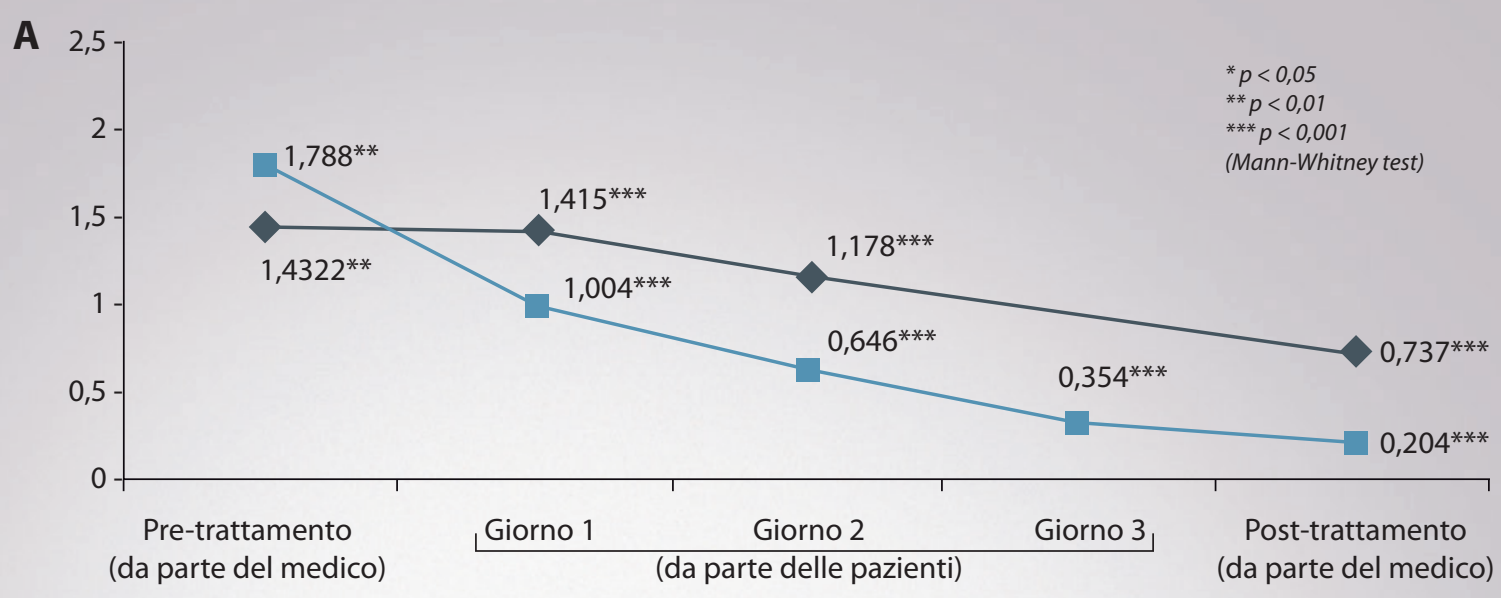

- Gruppo in trattamento

B

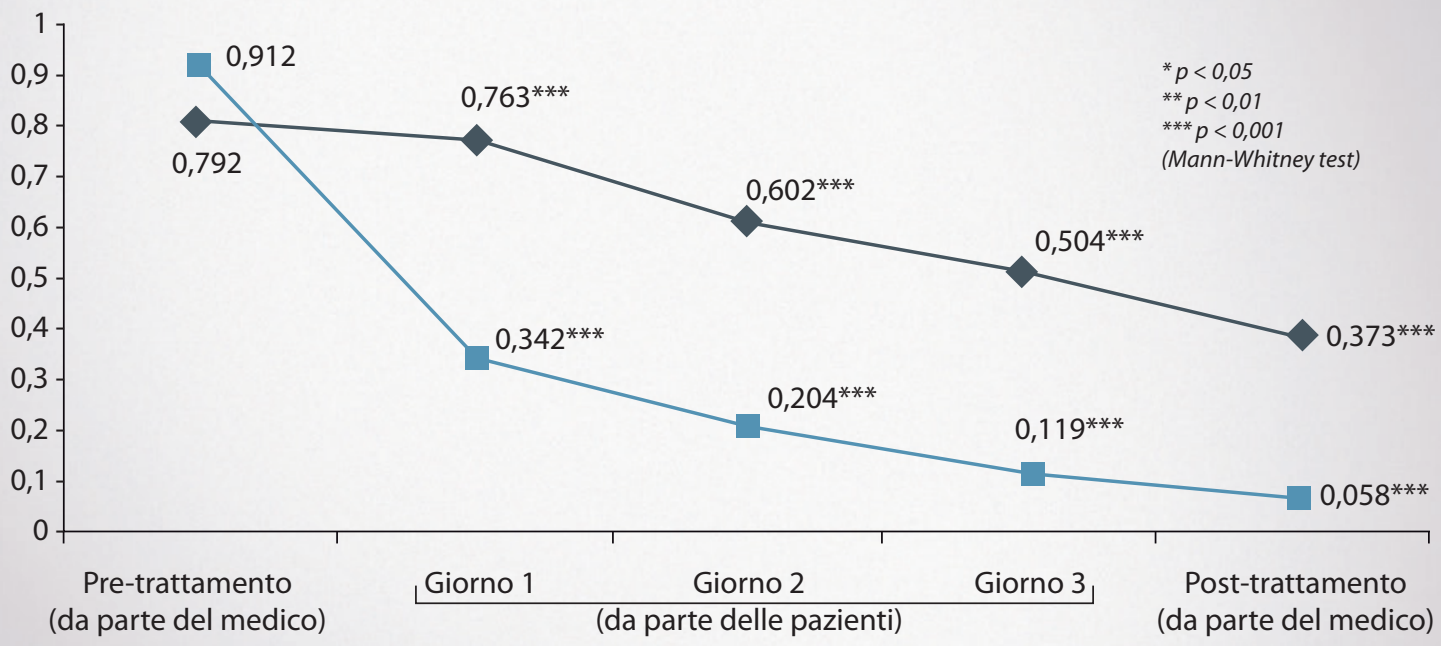

Gruppo in trattamento

Gruppo di controllo

Figura 5. Valutazione media della sensazione di bruciore $(A)$ e del prurito $(B)$ da parte del medico e delle pazienti nel gruppo in trattamento $(n=260)$ e nel gruppo di controllo $(n=236)$ [modificata da (58)]. 
soprattutto alle sue contestuali attività analgesica e antinfiammatoria.

In virtù della sua attività multiforme e polivalente, benzidamina è stata impiegata in ambito ginecologico, imponendosi come trattamento caratterizzato da un alto grado di appropriatezza nelle pazienti affette da vulvovaginiti di origine infettiva e non infettiva, in grado di garantire un rapido sollievo dai sintomi, dall'infiammazione e di controllare la proliferazione degli organismi patogeni. Nell'area cervicovaginale sottoposta ai danni da irradiazione terapeutica, benzidamina ha mostrato un rapido sollievo dalla sintomatologia e una rapida cicatrizzazione, prevenendo la necrosi post-operatoria dei tessuti vaginali.

\section{Disclosures}

L'autore dichiara di non avere conflitto d'interesse inerente all'articolo.

\section{Bibliografia}

1. Giné L, Ventín M, Petit J, Balagueró L. Study of benzydamine therapy of non-specific vaginitis. In: Genazzani AR, Silvestrini B (Eds). Benzydamine topical therapy in gynecology. Lancashire (UK): The Parthenon Publishing Group, 1988

2. Guaschino S, De Seta F, Piccoli M, et al. Aetiology of preterm labour: bacterial vaginosis. BJOG 2006;113(Suppl 3):46-51

3. Eckert LO. Clinical practice. Acute vulvovaginitis. N Engl J Med 2006;355(12):1244-52

4. Martín R, Soberón N, Vázquez F, Suárez JE. Vaginal microbiota: composition, protective role, associated pathologies, and therapeutic perspectives. Enferm Infecc Microbiol Clin 2008;26(3):160-7

5. Mårdh PA. The vaginal ecosystem. Am J Obstet Gynecol 1991;165(4 Pt 2):1163-8

6. Kaewsrichan J, Peeyananjarassri K, Kongprasertkit J. Selection and identification of anaerobic lactobacilli producing inhibitory compounds against vaginal pathogens. FEMS Immunol Med Microbiol 2006;48(1):75-83

7. Rönnqvist PD, Forsgren-Brusk UB, Grahn-Håkansson EE. Lactobacilli in the female genital tract in relation to other genital microbes and vaginal pH. Acta Obstet Gynecol Scand 2006;85(6):726-35

8. Lepargneur JP, Rousseau V. Protective role of the Doderlein flora. J Gynecol Obstet Biol Reprod (Paris) 2002;31(5):485-94

9. Aslim B, Kilic E. Some probiotic properties of vaginal lactobacilli isolated from healthy women. Jpn J Infect Dis 2006;59(4):249-53

10. Redondo-Lopez V, Cook RL, Sobel JD. Emerging role of lactobacilli in the control and maintenance of the vaginal bacterial microflora. Rev Infect Dis 1990;12(5):856-72

11. Pellis V, De Seta F, Crovella S, et al. Mannose binding lectin and C3 act as recognition molecules for infectious agents in the va- gina. Clin Exp Immunol 2005;139(1):120-6

12. Zara C, Montanari D, Polatti F. Morfologia submicroscopica di cellule di "Candida albicans" dopo esposizione alla benzidamina. In: Fioretti P, Silvestrini B, Esposito G (Eds). La terapia topica in ginecologica. 2nd Symposium on Topical Therapy in Gynaecology, Rome, 26 November, 1986. Milano: Società Milanese di Studi Ginecologici, 1987:13-8

13. Magliano EM, Curti C, Clerici P, Laurita L. Influenza del trattamento topico non specifico sull'equilibrio del microbiota vaginale. In: Guaschino S, Concia E, Filice C (a cura di). Le infezioni in ostetricia e ginecologia. Le malattie a trasmissione sessuale negli anni '80. Bologna: Monduzzi Editore, 1986

14. Carr PL, Felsenstein D, Friedman RH. Evaluation and management of vaginitis. J Gen Intern Med 1998;13(5):335-46

15. Owen MK, Clenney TL. Management of vaginitis. Am Fam Physician 2004;70(11):2125-32

16. Lévy G. Value of benzydamine, the first anti-inflammatory vaginal solution. Rev Fr Gynecol Obstet 1989;84(11):779-81

17. Montemagno U, Colace G, Piccili R, et al. Topical therapy of senile vaginitis. In: Genazzani AR, Silvestrini B (Eds). Benzydamine topical therapy in gynecology. Lancashire (UK): The Parthenon Publishing Group, 1988

18. Volterrani F, Tana S, Trenti N. Topical benzydamine in the treatment of vaginal radiomucositis. Int J Tissue React 1987;9(2):169-71

19. Kanaev SV, Baranov SB. [The use of Tantum Rosa in the prevention and treatment of radiation-induced vaginitis and proctitis] Vopr Onkol 1998;44(6):722-3

20. Silvestrini B. Benzydamine: a preclinical overview. In: Genazzani AR, Silvestrini B (Eds). Benzydamine topical therapy in gynecology. Lancashire (UK): The Parthenon Publishing Group, 1988

21. Cioli V, Corradino C, Scorza Barcellona P. Review of pharmacological data on benzydamine. Int J Tissue React 1985;7(3):205-13

22. Sironi M, Massimiliano L, Transidico P, et al. Differential effect of benzydamine on pro- versus anti-inflammatory cytokine production: lack of inhibition of interleukin-10 and interleukin-1 receptor antagonist. Int J Clin Lab Res 2000;30(1):17-9

23. Sironi M, Pozzi P, Polentarutti N, et al. Inhibition of inflammatory cytokine production and protection against endotoxin toxicity by benzydamine. Cytokine 1996;8(9):710-6

24. Griswold DE, Hillegass LM, Breton JJ, et al. Differentiation in vivo of classical non steroidal antiinflammatory drugs from cytokine suppressive antiinflammatory drugs and other pharmacological classes using mouse tumor necrosis factor alpha production. Drugs Exp Clin Res 1993;19(6):243-8

25. Sironi M, Milanese C, Vecchi A, et al. Benzydamine inhibits the release of tumor necrosis factor-alpha and monocyte chemotactic protein-1 by Candida albicans-stimulated human peripheral blood cells. Int J Clin Lab Res 1997;27(2):118-22

26. Riboldi E, Frascaroli G, Transidico P, et al. Benzydamine inhibits monocyte migration and MAPK activation induced by chemotactic agonists. Br J Pharmacol 2003;140(2):377-83 
27. Mathivanan S, de la Torre-Martinez R, Wolf C, et al. Effect of econazole and benzydamine on sensory neurons in culture. J Physiol Pharmacol 2016;67(6):851-8

28. Tagliapietra L. Action of $\mathrm{pH}$ on the antimicrobial effects of benzidamine. Boll Chim Farm 1970;109(6):374-9

29. Pina-Vaz C, Rodrigues AG, Sansonetty F, et al. Antifungal activity of local anesthetics against Candida species. Infect Dis Obstet Gynecol 2000;8(3-4):124-37

30. Magliano EM, Conti M, Clerici P, Laurita L. The role of benzydamine in the topical treatment of the so-called non-specific vaginitis. Int J Tissue React 1987;9(2):151-6

31. Baldock GA, Brodie RR, Chasseaud LF, et al. Pharmacokinetics of benzydamine after intravenous, oral, and topical doses to human subjects. Biopharm Drug Dispos 1991;12(7):481-92

32. Catanese B, Facchini V, Barillari G, Putzolu S. Serum levels of benzydamine following the topical use of this drug in gynecology. Clin Exp Obstet Gynecol 1980;7(2):84-8

33. Chasseaud LF, Catanese B. Pharmacokinetics of benzydamine. Int J Tissue React 1985;7(3):195-204

34. Maamer M, Aurousseau M, Colau JC. Concentration of benzydamine in vaginal mucosa following local application: an experimental and clinical study. Int J Tissue React 1987;9(2):135-45

35. Störmer E, Roots I Brockmöller J, et al. Benzydamine N-oxidation as an index reaction reflecting FMO activity in human liver microsomes and impact of FMO3 polimorphisms on enzyme activity. Br J Clin Pharmacol 2000;50(6):553-61

36. Lang DH, Rettie AE. In vitro evaluation of potential in vivo probes for human flavin-containing mono-oxygenase (FMO): metabolism of benzydamine and caffeine by FMO and P450 isoforms. Br J Clin Pharmacol 2000;50(4):311-4

37. Martella E, Balbi C, et al. Terapia topica delle flogosi vaginali in gravidanza. In: La terapia topica in ginecologia 1987;33-8

38. Capitanio GL, Lamba Doria L, Centonze L, et al. Vagino-cervical disinfection before chorionic villus sampling. Cervix Low Female Genital Tract 1987;5:151-5

39. Malinas Y, Favier M. Interete de l'Olpagyne, solution vaginale anti-inflammatoire dans la therapeutique fonctionnelle des vaginites. Prat Med 1987;23:40-2

40. Marcolin D, Tessari G, Sabino L, et al. The importance of benzydamine in the complementary therapy of vaginal trichomoniasis. Clin Exp Obstet Gynecol 1979;6(1 Suppl):50-3

41. Berrebi A, Pontonnier G, Reme JM. Treatment of functional symptoms of vaginal inflammation in vaginitis using benzydamine chlorhydrate. Rev Fr Gynecol Obstet 1986;81(10):567-9

42. Balsamo SB. The efficacy of concurrent treatment with benzydamine hydrochloride in vulvovaginitis. J Bras Ginec 1987;97(10):565-8

43. Quereux M. Clinical trial of a vaginal antiinflammatory solution (1\% benzydamine). Lille Med 1987;27(10):506-8

44. Frateschi M, Viganò R, Ossi C. Vaginiti aspecifiche recidivanti: proposta terapeutica. Giorn It Gin 1990;8:571-4
45. Calestani V, Cavalli C, Bonini R. In tema di terapia topica delle vaginiti aspecifiche: studio comparativo tra soluzioni di benzidamina e iodiopovidone. Giorn It Ost Gin 1987;5:441-6

46. Boselli F, Volpe A, Genazzani AR. Valutazione di alcuni farmaci d'impiego topico nella patologia cervico-vaginale. In: Fioretti P, Silvestrini B, Esposito G (Eds). La terapia topica in ginecologia. 2nd Symposium on Topical Therapy in Gynaecology, Rome, 26 November, 1986. Milano: Società Milanese di Studi Ginecologici, 1987:63-7

47. Ventolini G, Villa I, Guerra A. A controlled clinical study on the efficacy of benzydamine in the topical treatment of non-specific cervicitis and vaginitis. Int J Tissue React 1987;9(2):157-67

48. Romanini C, Tiriduzzi M, Manso E, et al. Comparison between the activities of benzydamine and metronidazole in the therapy of bacterial vaginosis. In: Fioretti P, Silvestrini B, Esposito G (Eds). La terapia topica in ginecologica. 2nd Symposium on Topical Therapy in Gynaecology, Rome, 26 November, 1986. Milano: Società Milanese di Studi Ginecologici, 1987:35-45

49. Brémond A. Clinical evaluation of benzydamine for treatment of vaginitis: results of a randomized study with emphasis on functional symptoms. Int J Tissue React 1987;9(2):147-9

50. Simon FT. Topical use of benzydamine in ginecology. Folha Med 1982;85(5-6):971-4

51. Brémond A, Andrè D, Ducret L, et al. Treatment of vaginitis with benzydamine: preliminary results of a randomized study. Int $\mathrm{J}$ Tissue React 1985;7(3):225-7

52. Monfort Monfort M, Martin-Loeches de la Lastra M, Richard Rocamora MA. Estudio de la evolucion de las cervicovaginitis en function del tratamiento. Semer 1993;19(1):31-9

53. De Virgiliis G, Stillo A, Crippa L, et al. Cervico-vaginitis and contraception. Efficacy of a benzydamine vaginal solution. Satellite Symposium of the 64th S.I.G.O. National Congress Rome November 26, 1986

54. Villalba J, Estevez J, Villa PJ. The role of topical benzydamine in vaginal pessary users. Cienc Ginecol 2001;5(2):83-7

55. Bentivoglio G, Diani F. Use of topical benzydamine in gynecology. Clin Exp Obstet Gynecol 1981;8(3):103-10

56. Wolf JK. Prevention and treatment of vaginal stenosis resulting from pelvic radiation therapy. Commun Oncol 2006;3:665-71

57. Chulkova OV, Demidova LV, Udovichenko VI. Findings obtained by using Tantum Rosa product in the Clinical experience with the use of Tantum Rosa in cancer patients. Vopr Onkol 1997;43(3):339-40

58. Czaika R, Torbè A. Assessment of the efficacy of benzydamine (Tantum Rosa) in form of vaginal lavage in the treatment of perineal complaints in the early puerperium Pol J Gynecol Invest 2001;4(2):99-103

59. Caranti S. Periodic Safety Update Report (PSUR) TANTUM ROSA - Benzydamine for gynaecological use (period covered January 1st, 2005 - August 31st, 2008. Pharmacovigilance Service of ACRAF SpA, Rome, Italy 